\title{
Very High Resolution Spaceborne SAR Tomography in Urban Environment
}

\author{
Xiao Xiang Zhu, Student Member, IEEE, and Richard Bamler, Fellow, IEEE
}

\begin{abstract}
Synthetic aperture radar tomography (TomoSAR) extends the synthetic aperture principle into the elevation direction for 3-D imaging. It uses stacks of several acquisitions from slightly different viewing angles (the elevation aperture) to reconstruct the reflectivity function along the elevation direction by means of spectral analysis for every azimuth-range pixel. The new class of meter-resolution spaceborne SAR systems (TerraSAR-X and COSMO-Skymed) offers a tremendous improvement in tomographic reconstruction of urban areas and man-made infrastructure. The high resolution fits well to the inherent scale of buildings (floor height, distance of windows, etc.). This paper demonstrates the tomographic potential of these SARs and the achievable quality on the basis of TerraSAR-X spotlight data of urban environment. A new Wiener-type regularization to the singular-value decomposition method-equivalent to a maximum a posteriori estimator-for TomoSAR is introduced and is extended to the differential case (4-D, i.e., space-time). Different model selection schemes for the estimation of the number of scatterers in a resolution cell are compared and proven to be applicable in practice. Two parametric estimation algorithms of the scatterers' elevation and their velocities are evaluated. First 3-D and 4-D reconstructions of an entire building complex (including its radar reflectivity) with very high level of detail from spaceborne SAR data by pixelwise TomoSAR are presented.
\end{abstract}

Index Terms-Differential synthetic aperture radar tomography (D-TomoSAR), spotlight SAR, TerraSAR-X, urban mapping.

\section{INTRODUCTION}

A CONVENTIONAL space- or airborne synthetic aperture radar (SAR) maps the 3-D reflectivity distribution of a scene to be imaged into the 2-D azimuth-range $(x-r)$ plane. This can be seen as a projection along the third radar coordinate, namely, elevation $(s) . x, r$, and $s$ form an orthogonal coordinate system specific to the particular SAR imaging geometry. This projection particularly handicaps the interpretation of SAR images of the following: 1) volumetric scatterers and 2) urban areas and man-made objects, i.e., objects with constructive elements oriented at steeper angles than the local incidence angle.

Manuscript received April 29, 2009; revised December 15, 2009 and March 25, 2010. Date of publication June 28, 2010; date of current version November 24, 2010.

$\mathrm{X}$. Zhu is with the Lehrstuhl für Methodik der Fernerkundung, Technische Universität München, 80333 Munich, Germany (e-mail: xiaoxiang. zhu@bv.tum.de).

R. Bamler is with the Remote Sensing Technology Institute (IMF), German Aerospace Center (DLR), 82234 Wessling, Germany, and also with the Lehrstuhl für Methodik der Fernerkundung, Technische Universität München, 80333 Munich, Germany.

Digital Object Identifier 10.1109/TGRS.2010.2050487

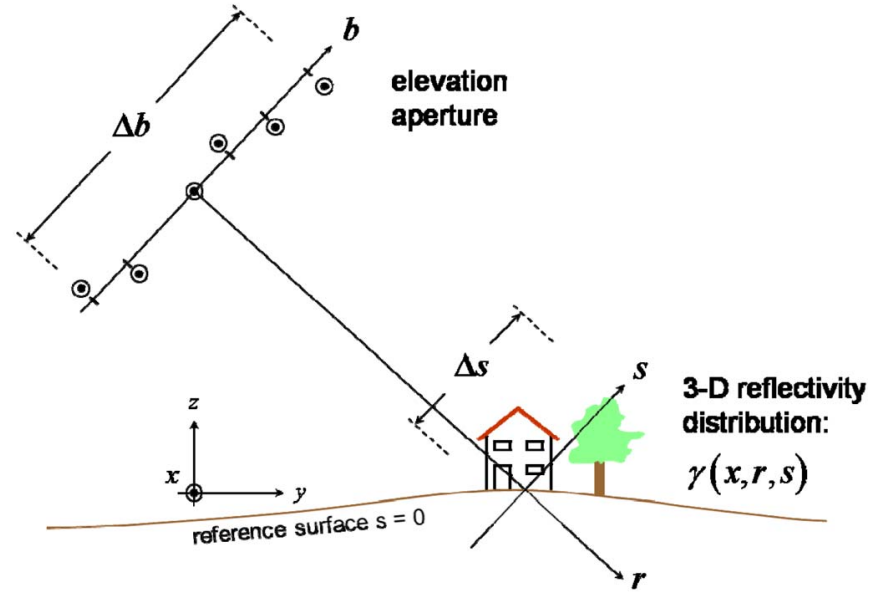

Fig. 1. TomoSAR imaging geometry. The coordinate $\mathrm{s}$ is referred to as elevation.

SAR tomography (TomoSAR), introduced to SAR in the early 1990s [1], extends the synthetic aperture principle into the elevation direction for 3-D imaging (within the first Born approximation). It uses data stacks of several acquisitions from slightly different viewing angles (the elevation aperture) to reconstruct the reflectivity function along the elevation direction by means of spectral analysis for every azimuth-range pixel, and hence obtains focused 3-D SAR images (Fig. 1). A further extension is differential SAR tomography (D-TomoSAR) [2], also referred to as 4-D focusing. It provides retrieval of both the elevation and the deformation information of multiple scatterers inside an azimuth-range resolution cell and therefore obtains a 4-D (space-time) map of scatterers.

Compared to computed axial tomography, known from medical imaging, TomoSAR uses only a small angular diversity. Hence, instead of back projection, spectral estimation is sufficient for TomoSAR if the range migration $\delta r$ caused by the different viewing angles is much smaller than the range resolution $\rho_{r}$. This gives a limitation to the extent $\Delta s$ of the illuminated objects

$$
\Delta s \ll \frac{\rho_{r} r}{\Delta b}
$$

where $r$ is the range and $\Delta b$ is the perpendicular (or effective) baseline range (i.e., the elevation aperture length). The term "baseline" is a heritage of interferometry. It is the spatial dimension in the elevation aperture, relative to a reference (master) track.

The first experiments in TomoSAR were carried out in the laboratory [3] under ideal experimental conditions or by using 
airborne systems [1], [4]. Spaceborne TomoSAR tests were reported in [5] and [6]. It has been applied to C-band European Remote Sensing Satellite (ERS) data over extended scenes in [7] and to TerraSAR-X data in [8]. In [9], the single- and double-scatterer cases were separated. The concept of 4-D SAR imaging (D-TomoSAR) was proposed in [2] and first applied to ERS data in [10].

The major challenges in spaceborne TomoSAR are the following. First, acquisitions are unevenly distributed in baseline, i.e., in the elevation aperture space, so that appropriate regularization is required instead of a classical Fourier-based inversion [11]. Second, 3-D data cannot be collected simultaneously, at least with existing satellites, but must rather be acquired via repeated passes that are separated in time. Hence, timedependent phase terms from motion and from the propagation medium are present in the data and must be considered. Third, the number of acquisitions may be limited.

In 2007, SAR remote sensing from space made a big leap forward; the German TerraSAR-X and the Italian COSMOSkymed satellites have been launched. They deliver SAR data with a very high spatial resolution of up to $1 \mathrm{~m}$ compared to medium (10-30-m)- and high (3-10-m)-resolution SAR systems available so far. The advantage of very high resolution (VHR) imagery for cartographic applications is obvious. The real potential of this class of SAR data, however, lies in applications, where the coherent nature of SAR data is exploited, like interferometry or tomography. The $1-\mathrm{m}$ resolution is particularly helpful when it comes to 2-D, 3-D, or 4-D imaging of buildings and urban structures. The inherent spatial scales of buildings are dominated by the typical height between floors of 3-3.5 m, i.e., in slant range (at 30 $) 2.6-3.0 \mathrm{~m}$, and the distance of windows. Hence, for imaging of urban structures, we can expect a tremendous improvement in information content when we go from high to VHR. We work with TerraSAR-X spotlight data. These VHR X-band spaceborne repeat-pass tomographic data stacks of urban areas have some particular properties: A very detailed view of individual buildings is possible; the density of bright (high clutter-to-noise ratio) points, like persistent scatterers, is extremely high $\left(40000-100000 / \mathrm{km}^{2}\right)$. However, also nonlinear (e.g., thermally induced) movements of different building parts must be expected and will introduce additional phase errors and require robust inversion methods.

This paper aims at demonstrating the potential of the new class of VHR spaceborne SAR systems for TomoSAR in urban environment. In particular, we introduce a new Wienertype regularization to the singular-value decomposition (SVD) method [7] for TomoSAR and extend it to the D-TomoSAR case (Section IV). Different model selection schemes for the estimation of the number of scatterers are evaluated and validated (Section V). Two parametric estimation algorithms of the scatterers' elevation and their velocities are evaluated (Section VI). We demonstrate first 3-D and 4-D reconstructions of an entire building from spaceborne VHR data by pixelwise TomoSAR (Section VII). We will concentrate on urban areas and manmade infrastructure. Volumetric objects, like trees, can be considered incoherent in X-band repeat pass and are hence treated as noise. A limited number (typically one to three) of scatterers is expected along every elevation profile that allows parametric estimation.
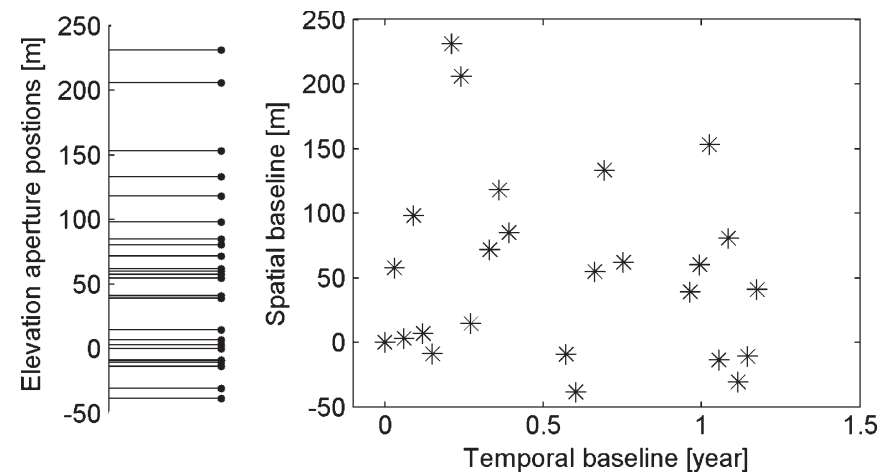

Fig. 2. (Left) Elevation aperture sampling positions of the 25 acquisitions (Right) Spatial-temporal baseline distribution (to be used for D-TomoSAR in Section III).

\section{DATA SET}

For the purpose of this paper, we work with TerraSAR-X "high-resolution spotlight data" (TerraSAR-X product terminology) acquired with a range bandwidth of $300 \mathrm{MHz}$. They have a slant-range resolution of $0.6 \mathrm{~m}$ and an azimuth resolution of $1.1 \mathrm{~m}$. In this mode, image lengths of $5-10 \mathrm{~km}$ can be acquired, which is sufficient for our investigations in urban environments. Note that interferometric use of spotlight data requires some special care, e.g., in synchronous data acquisition, coregistration, and resampling [12]. Our test site is Las Vegas, NV, U.S. The acquisition repeat cycle is 11 days. The orbit of TerraSAR-X is controlled in a predefined tube of $500 \mathrm{~m}$ diameter throughout the entire mission [13]. Due to this small orbit tube, the precondition for the spectral estimation approximation mentioned in (1) is very easily fulfilled. It is worth mentioning that, unlike in airborne TomoSAR, the relatively large temporal separation of the repeated passes of spaceborne data collection introduces motion and atmospheric phase contributions that have to be accounted for-albeit as nuisance parameters. This requires a lot more data sets to get unambiguous results and resolve multiple scatterers inside an azimuth-range cell. In our experiment, a data stack of 25 scenes is used for our test site. The elevation aperture sampling positions are shown in Fig. 2. The elevation aperture size $\Delta b$ is about $269.5 \mathrm{~m}$. According to (1), with $r=704 \mathrm{~km}$ and $\rho_{r}=0.6 \mathrm{~m}$, the elevation extent $\Delta s$ of the illuminated objects must be much smaller than $1568 \mathrm{~m}$. This is always true for our test site. Therefore, in the following sections, we handle TomoSAR as a spectral estimation problem, and the detailed system model will be introduced in Section III.

For nonparametric spectral analysis, the expected elevation resolution $\rho_{s}$, i.e., the width of the elevation point response function (PRF), depends on the elevation aperture length $\Delta b$ and is approximately (sufficiently dense sampling of the elevation aperture provided)

$$
\rho_{s}=\frac{\lambda r}{2 \Delta b}
$$

where $\lambda$ is the wavelength. It results in $40.5-\mathrm{m}$ resolution in elevation expected for our stack, which is approximately $20-\mathrm{m}$ resolution in height with the elevation-to-height factor $\sin \theta$, where $\theta$ is the incidence angle and equals $31.8^{\circ}$ here. This, however, does not mean that individual scatterers can only be 


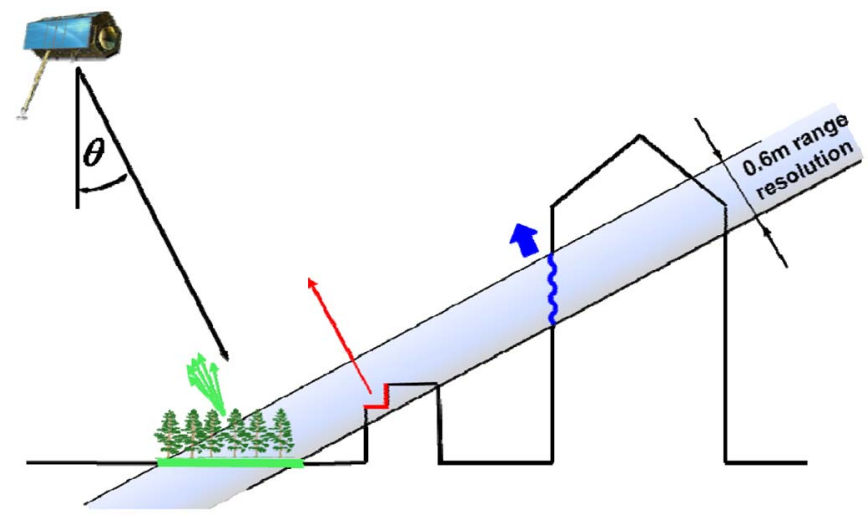

Fig. 3. Possible signal contributions in a single SAR image azimuth-range pixel.

located to within this poor elevation resolution. The CramérRao lower bound (CRLB) on elevation estimates can be shown to be [14]

$$
\sigma_{\hat{s}}=\frac{\lambda r}{4 \pi \sqrt{N O A} \cdot \sqrt{2 S N R} \cdot \sigma_{b}}
$$

where NOA is the number of acquisitions, SNR is the signalto-noise ratio, and $\sigma_{b}$ is the standard deviation of the baseline distribution. For instance, the stack used in this example has $\sigma_{b}=70.9 \mathrm{~m}$. For many bright points, we can assume an SNR of $10 \mathrm{~dB}$; then, the CRLB on elevation estimation is $1.1 \mathrm{~m}$, i.e., almost a $1 / 40$ of the elevation resolution.

\section{TOMOSAR}

\section{A. System Model}

For a single SAR acquisition, the focused complex-valued measurement $g_{n}\left(x_{0}, r_{0}\right)$ of a specific azimuth-range pixel $\left(x_{0}, r_{0}\right)$ for the $n$th acquisition with aperture position $b_{n}$ and temporal baseline $t_{n}$ is the integral of the reflected signal along the elevation direction, as shown in Fig. 3. In VHR X-band data, we expect the following signal contributions (see Fig. 3).

1) Weak diffuse scattering from-mostly horizontal or vertical—rough surfaces (roads and building walls). They have an elevation extent of $\rho_{r} / \tan \theta$ for horizontal and $\rho_{r} \cdot \tan \theta$ for vertical surfaces. In both cases, these extents are much smaller than our elevation resolution $\rho_{s}$, and hence, these surfaces can be treated as discrete scatterers in the elevation direction (delta functions).

2) Strong returns from metallic structures or specular and dihedral or trihedral reflections. These are points that would also be used in persistent scatter interferometry. They are the dominating signal contributions. With VHR SAR data, the density of these points can be very high, as mentioned before.

3) Returns from volumetric scatterers, e.g., from vegetation. These result in a continuous signal background in elevation. These ensembles of scatterers, however, often decorrelate in time, and their response is therefore treated as noise.

The noise sources are the following.

a) Gaussian noise, which is caused by thermal noise and temporal decorrelation, as mentioned previously. b) Calibration errors in amplitude. According to an unpublished DLR internal calibration report [15], the radiometric stability of TerraSAR-X, i.e., the amplitude variations within one stack, is $0.14 \mathrm{~dB}$ and is therefore negligible compared to our typical SNR.

c) Phase errors caused by atmospheric delay and unmodeled motion. They require robust and phase-error-tolerant estimation methods.

One SAR acquisition may be considered to be one tomographic projection of the complex reflectivity of the object along elevation [16] (note that the deformation term is ignored here for simplicity)

$$
g_{n}=\int_{\Delta s} \gamma(s) \exp \left(-j 2 \pi \xi_{n} s\right) d s, \quad n=1, \ldots, N
$$

where $\gamma(s)$ represents the reflectivity function along elevation $s . \xi_{n}=-2 b_{n} /(\lambda r)$ is the spatial (elevation) frequency. The continuous-space system model of (4) can be approximated by discretizing the continuous reflectivity function along $s$ within its extent $\Delta s$ by $s_{l}(l=1, \ldots, L)$

$$
g_{n} \approx \delta s \cdot \sum_{l=1}^{L} \gamma\left(s_{l}\right) \exp \left(-j 2 \pi \xi_{n} s_{l}\right), \quad n=1, \ldots, N
$$

where $L$ is the number of discrete elevation indices and the discretization interval is $\delta s=\Delta s /(L-1)$. After dropping the inconsequential leading constant $\delta s$, the system imaging model becomes

$$
\mathrm{g}=\mathbf{R} \gamma
$$

where $\mathbf{g}$ is the measurement vector with $N$ elements $g_{n}, \mathbf{R}$ is an $N \times L$ mapping matrix with $R_{n l}=\exp \left(-j 2 \pi \xi_{n} s_{l}\right)$, and $\gamma$ is the discrete reflectivity vector with $L$ elements $\gamma_{l}=\gamma\left(s_{l}\right)$. Equation (6) is essentially an irregularly sampled discrete Fourier transform of the elevation profile $\gamma(s)$. The objective of TomoSAR is to retrieve the reflectivity profile for each azimuth-range pixel and then use it to estimate scattering parameters such as the number of scatterers present in the cell, their elevations, reflectivities, and line-of-sight (LOS) deformation velocities (see Section IV). This can be achieved from a spectral analysis of the multipass data stack with $N$ SAR acquisitions.

\section{B. Processing Sequence}

The processing procedure, with the objective of reconstructing the 3-D scatterer distribution from measurements of the scattered field and estimating LOS deformation, is shown in Fig. 4. The preprocessing, including atmospheric phase screen correction, is performed by the German Aerospace Center (DLR)'s PSI-GENESIS system [17]-[20].

To acquire an estimate of the reflectivity profile along elevation for a certain azimuth-range pixel, nonparametric spectral analysis is used for the first stage of processing. Except the maximum elevation extent $\Delta s$ of the object and some statistical properties of the prior and the noise, no prior 


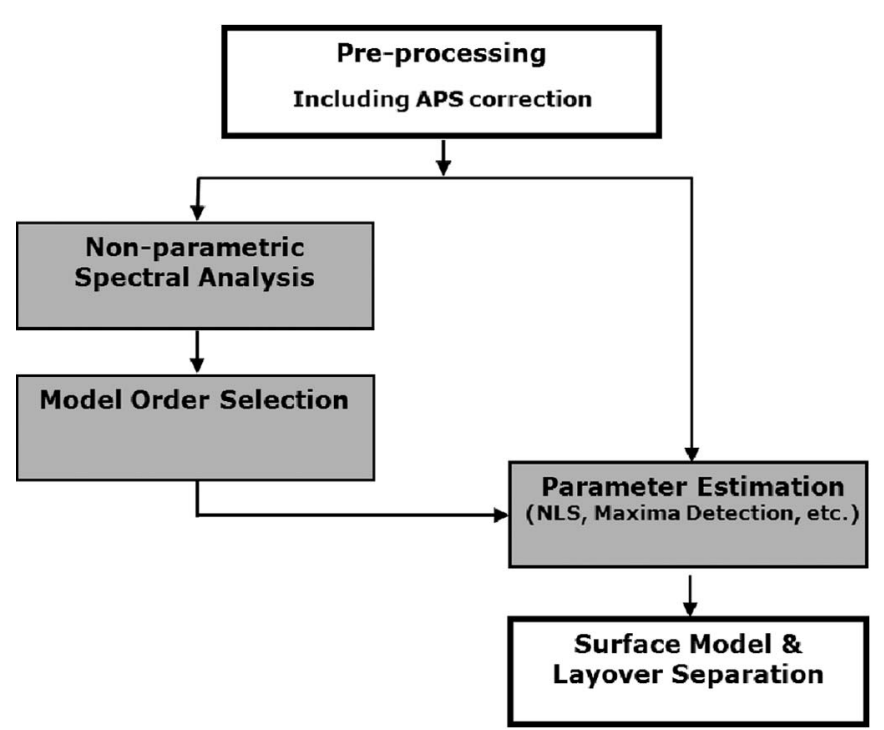

Fig. 4. Processing sequence of tomographic SAR data stacks.

knowledge, such as the number of scattering objects, and no assumption about the scattering mechanism are required. The estimated profile is then used as a prior knowledge for model order selection and parameter estimation. There are many inversion methods, such as SVD, Capon, multiple signal classification (MUSIC), etc. However, it is not our purpose to compare different spectral estimation methods, which have been discussed in detail in [21]-[23]. The high phase errors due to unmodeled motion require robust methods (see selfcancellation of Capon [24]). In addition, we want to maintain the full range and azimuth resolution since the 1-m rangeazimuth resolution of the data is essential for urban applications. As mentioned in Section I, the inner scale of buildings is typically in the range of 2-3 m. Any blurring, as it is necessary for the covariance matrix estimation in MUSIC, Capon, etc., is lethal for the information content; individual bright points will be merged to bright clusters. Moreover, the typical signals of buildings are not ergodic. Taking all this into account, the SVD-based method has been chosen because of its good behavior at high noise levels without compromising azimuth-range resolution. With nonparametric estimates, the scatterers' distribution in the elevation-velocity $(v-s)$ plane, to be introduced later, and a 3-D reflectivity map of the entire illuminated scene are obtained. They are input to model order selection, i.e., to the estimation of the number of discrete (pointlike) scatterers.

With prior knowledge of the number of scatterers, a parametric spectral estimator, for instance, nonlinear least squares (NLS), which is the maximum-likelihood estimator (MLE) for Gaussian white noise, can be applied to the measurements to refine the estimates at the cost of large computational effort. Alternatively, we can simply estimate the location of the scatterers by detecting the peaks of the nonparametric SVD estimates, which is much faster but may introduce estimation bias caused by interference between multiple scatterers.

We will not go into the details of preprocessing as they are sufficiently addressed in the literature. The remaining modules (gray boxes in Fig. 4) are outlined next.

\section{MAP ESTIMATOR AND WIENER SVD}

The standard MAP estimator for $\gamma$ from (6) is given by

$$
\hat{\gamma}_{\mathrm{MAP}}=\left(\mathbf{R}^{\mathrm{T}} \mathbf{C}_{\varepsilon \varepsilon}^{-1} \mathbf{R}+\mathbf{C}_{\gamma \gamma}^{-1}\right)^{-1} \mathbf{R}^{\mathrm{T}} \mathbf{C}_{\varepsilon \varepsilon}^{-1} \mathbf{g}
$$

where $\mathbf{C}_{\varepsilon \varepsilon}$ is the noise covariance matrix and $\mathbf{C}_{\gamma \gamma}$ is the covariance matrix of the prior. It reduces to

$$
\hat{\gamma}_{\mathrm{MAP}}=\left(\mathbf{R}^{\mathrm{T}} \mathbf{R}+|\varepsilon|^{2} \mathbf{I}\right)^{-1} \mathbf{R}^{\mathrm{T}} \mathbf{g}
$$

if both the noise and the prior are assumed to be white, i.e., the noise covariance matrix $\mathbf{C}_{\varepsilon \varepsilon}=|\varepsilon|^{2} \mathbf{I}$, the covariance matrix of the prior $\mathbf{C}_{\gamma \gamma}=\mathbf{I}$, and the signal power is assumed to be normalized to unity. This nonparametric spectral estimation method has been chosen because of its robustness at high noise levels without sacrificing the azimuth-range resolution.

Although the MAP estimator from (8) could be implemented directly, the treatment of the problem in the singular-value (SV) space is helpful. As will be shown, the distribution of the SVs helps us understand the determinedness of the problem and estimate the noise level.

In this section, we will show that the MAP estimator is equivalent to a Wiener-type regularization of the SVs. It is a more strict solution than the original truncated SVD (TSVD) method [16]. We also give its extension to D-TomoSAR.

\section{A. SVD method}

The SVD inversion framework has been elegantly described in [16]. The discrete reflectivity signal $\gamma$ can be reconstructed from $\mathbf{g}$ through pseudoinversion of the imaging system matrix $\mathbf{R}$ [(6)]. However, due to the nonuniform track distribution, the solution may include significant noise propagation due to the ill-conditioned nature of the problem. The SVD is a simple and valuable tool for analyzing image quality and the amount of independent information about the unknowns that can be reliably retrieved from observations in the presence of noise [11]. The SVD of $\mathbf{R}$ is a decomposition of the form

$$
\mathbf{R}=\mathbf{U} \boldsymbol{\Sigma} \mathbf{V}^{\mathrm{T}}=\sum_{n=1}^{N} \mathbf{u}_{n} \sigma_{n} \mathbf{v}_{n}^{\mathrm{T}} .
$$

where $\mathbf{U}=\left(\mathbf{u}_{\mathbf{1}}, \ldots, \mathbf{u}_{\mathbf{N}}\right)$ and $\mathbf{V}=\left(\mathbf{v}_{\mathbf{1}}, \ldots, \mathbf{v}_{\mathbf{N}}\right)$ are matrices with orthonormal columns, $\mathbf{U}^{\mathbf{T}} \mathbf{U}=\mathbf{V}^{\mathbf{T}} \mathbf{V}=\mathbf{I}_{\mathbf{N}}$, and $\boldsymbol{\Sigma}=$ $\operatorname{diag}\left(\sigma_{1}, \ldots, \sigma_{N}\right)$ has nonnegative diagonal elements such that $\sigma_{1} \geq \cdots \geq \sigma_{N} \geq 0 . \sigma_{n}$ denotes the SVs of $\mathbf{R}$, while the vectors $\mathbf{u}_{n}$ and $\mathbf{v}_{n}$ are the left and right singular vectors of $\mathbf{R}$, respectively. Consider now that an estimate of $\gamma$ is obtained via the pseudoinverse $\mathbf{R}^{\dagger}$. Using the SVD, we get

$$
\hat{\boldsymbol{\gamma}}=\mathbf{R}^{\dagger} \mathbf{g}=\sum_{n=1}^{N} \sigma_{n}^{-1}\left(\mathbf{u}_{n}^{\mathrm{T}} \mathbf{g}\right) \mathbf{v}_{n}
$$

Due to the reciprocal of $\sigma_{n}$, noise propagation caused by small SVs will compromise this solution, and regularization tools are required. 


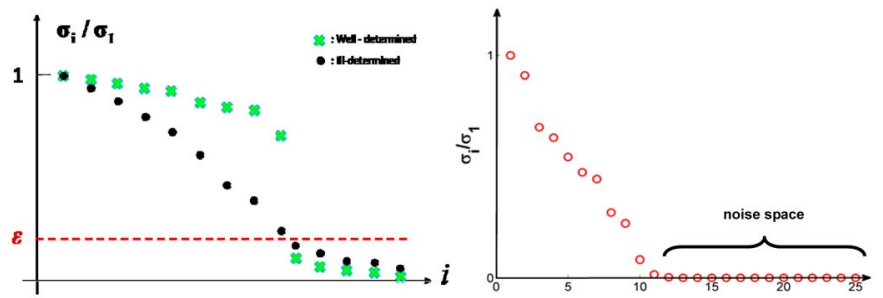

Fig. 5. (Left) SV spectra corresponding to ill-conditioned matrices with welland ill-determined numerical ranks. (Right) Corresponding SV spectrum of our TomoSAR configuration with elevation aperture sampling from Fig. 2.

\section{B. Wiener Regularization}

A well-known method for dealing with an ill-conditioned matrix in problem (7) is TSVD [25]. The basic idea of TSVD and other regularization methods is to impose additional requirements on the solution, thus hopefully damping the contributions from the errors of the right-hand side of (7). In the case of TSVD, this is achieved by discarding the components of the solution corresponding to the smallest $N-Q \mathrm{SVs}$. These contributions to the solution are most likely noise and would be amplified unduely by the inverse of $\sigma_{n} . Q$ is called "numerical rank" or "effective rank" of $\mathbf{R}$ defined by the number of SVs bigger than some noise level $\varepsilon$. The TSVD was implemented in [16] for an experiment with 44 acquisitions, and robust performance was achieved.

Depending on the SV spectrum, it is common to characterize an ill-conditioned matrix as either with a well- or an ill-determined numerical rank [26]. An ill-conditioned matrix with a well-determined numerical rank has a well-defined gap between the significant SVs contributing to the signal space and the small SVs contributing to the noise space (see Fig. 5, left, green crosses). Matrices with ill-determined rank degrade gradually from the signal to the noise space (see Fig. 5, left, black dots). Any distribution of SVs in between the two extremes of the left figure of Fig. 5 may, of course, be expected in practical applications. However, from the perturbation theory for the TSVD [25], TSVD is a stable method only for problems with a well-determined numerical rank.

The corresponding SVs of our Las Vegas TerraSAR-X data set with the baseline distribution of Fig. 2 are shown in the right plot of Fig. 5. It is obviously of ill-determined numerical rank. The result is then overly dependent on how a hard threshold is set. Transforming the MAP estimator of (8) to the SV space results readily in a soft thresholding, e.g., weighting the SVs according to their magnitudes, also referred to as a Tikhonov regularization

$$
\begin{aligned}
\hat{\boldsymbol{\gamma}}_{\mathrm{MAP}} & =\left(\boldsymbol{\Sigma}^{T} \boldsymbol{\Sigma}+|\varepsilon|^{2} \mathbf{I}\right)^{-1} \mathbf{V} \Sigma^{T} \mathbf{U}^{T} \mathbf{g} \\
& =\sum_{n=1}^{N} \sigma_{n, \text { Wiener }}^{-1}\left(\mathbf{u}_{n}^{T} \mathbf{g}\right) \mathbf{v}_{n}
\end{aligned}
$$

where $\sigma_{n, \text { Wiener }}^{-1}$ denotes the optimum weights

$$
\sigma_{n, \text { Wiener }}^{-1}=\frac{\sigma_{n}}{\left|\sigma_{n}\right|^{2}+|\varepsilon|^{2}} .
$$

It replaces $\sigma_{n}^{-1}$ in (10). $|\varepsilon|^{2}$ is the noise power level. A small $\varepsilon$ corresponds to a high SNR. This type of weighting resembles the Wiener filter under white noise, and hence, we call the
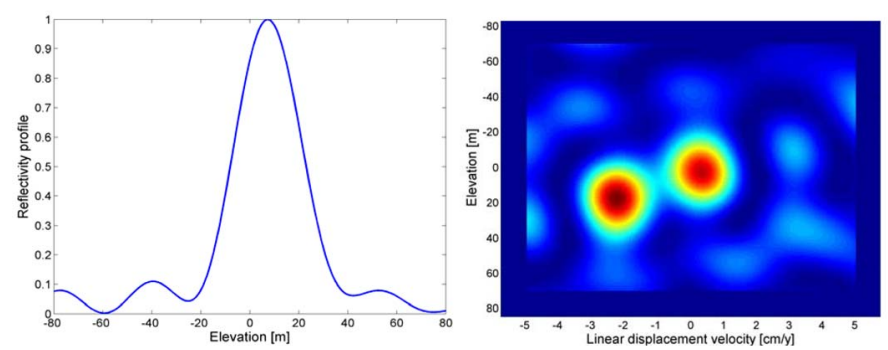

Fig. 6. Two close scatterers separable with D-TomoSAR due to the different velocities. (Left) Reconstructed reflectivity profile with TomoSAR; the scatterers are not separable. (Right) Retrieved scatterer distribution in the $s-v$ plane with D-TomoSAR.

method SVD-Wiener. It provides more stable performance, particularly for the case of few (15-30) acquisitions.

Now, we come to the problem of estimating the noise level $\varepsilon$. Let us define coefficients $\beta_{n}=\mathbf{u}_{\mathbf{n}}^{\mathbf{T}} \mathbf{g}$, which are the projection of measurements onto the singular vectors. The noise level can be estimated from the $n_{\varepsilon}$ coefficients $\beta_{n}\left(n=N-n_{\varepsilon}+\right.$ $1, \ldots, N)$ corresponding to the noise space (in our experiment, $n_{\varepsilon}$ can be set to 14; see Fig. 5, right). An estimate $\hat{\varepsilon}$ of the noise level $\varepsilon$ for every azimuth-range pixel can be obtained via

$$
\hat{\varepsilon}=\sqrt{\frac{N}{n_{\varepsilon}} \sum_{n=N-n_{\varepsilon}+1}^{N}\left|\beta_{n}\right|^{2} .}
$$

\section{Extension to D-TomoSAR}

Taking the motion term into account, the system model (4) can be extended to

$$
\begin{aligned}
g_{n}=\int_{\Delta s} \gamma(s) \exp \left(-j 2 \pi\left(\xi_{n} s+\eta_{n} V(s)\right)\right) d s, & \\
& n=1, \ldots, N
\end{aligned}
$$

where $V(s)$ is the deformation LOS velocity profile along elevation and $\eta_{n}=2 t_{n} / \lambda$ may, in analogy, be called a "velocity frequency." Formally, (14) can be rewritten as

$$
\begin{array}{r}
g_{n}=\int_{\Delta v} \int_{\Delta s} \gamma(s) \delta(v-V(s)) \exp \left(-j 2 \pi\left(\xi_{n} s+\eta_{n} v\right)\right) d s d v \\
n=1, \ldots, N \quad
\end{array}
$$

where $\Delta v$ is the velocity range, which is typically on the order of some tens of centimeters per year. Equation (12) is a 2-D Fourier transform of $\gamma(s) \delta(v-V(s))$, which is a delta line in the elevation-velocity $(s-v)$ plane along $v=V(s)$. Projected onto the elevation axis, $\gamma(s) \delta(v-V(s))$ follows the reflectivity profile $\gamma(s)$. If we accept $\gamma(s) \delta(v-V(s))$ as the object to be reconstructed, the SVD-based spectral estimation methods used for TomoSAR can be easily extended to the 4-D case that includes the LOS deformation terms, i.e., D-TomoSAR [2], [10]. Instead of treating the deformation phase term as noise, D-TomoSAR can provide retrieval of the elevation and deformation information of multiple scatterers inside an azimuth-range resolution cell and thus obtain a 4-D map of 
scatterers. It is required for reliable 3-D and 4-D city mapping from repeat-pass acquisitions.

Likewise, due to the ill-conditioning of the problem, regularization tools, such as TSVD and Wiener filtering, can be implemented, as described earlier. Fig. 6 shows an example. Two scatterers with an elevation distance of $20 \mathrm{~m}\left(s_{1}=0 \mathrm{~m} ; s_{2}=\right.$ $20 \mathrm{~m}$ ) are simulated. With standard 3-D TomoSAR, they are not separable with our baseline configuration since they are within a 40-m elevation resolution element, as shown in the left plot of Fig. 6. However, due to their different velocities $\left(V_{1}=\right.$ $0 \mathrm{~cm} /$ year; $V_{2}=-2 \mathrm{~cm} /$ year), they can be easily distinguished in the $s-v$ plane, as shown in the right panel of Fig. 6.

\section{Model Order Selection}

By implementing nonparametric spectral estimation, the reflected power along the elevation direction can be extracted. Model selection schemes aim at estimating the number of point scatterers along elevation inside an azimuth-range pixel [29].

Let $k$ be a parameter that defines the complexity of the model. In our case, $k$ is the number of parameters to describe $\gamma$. It depends on the number of scatterers $n_{p}$ in the azimuth-range pixel. As each scatterer can be described by three parameters (amplitude, phase, and elevation), $k=3 n_{p}$. Let further $\boldsymbol{\theta}(k)$ be the vector of the unknown amplitudes, phases, and elevations for all the $n_{p}$ scatterers. Then, the reflectivity profile can be written as $\boldsymbol{\gamma}(\boldsymbol{\theta}(k))$. The relationship between $\boldsymbol{\gamma}(\boldsymbol{\theta}(k))$ and the observed data $\mathbf{g}$ is described by the observation model [(6)]. $p(\mathbf{g} \mid \boldsymbol{\theta}(k), k)$ is the likelihood function. Under the assumption that the model errors or disturbances $\mathbf{e}=\mathbf{g}-\mathbf{R} \boldsymbol{\gamma}(\boldsymbol{\theta}(k))$ are circular Gaussian distributed with zero mean and a covariance matrix of $\mathbf{C}_{\varepsilon \varepsilon}=|\varepsilon|^{2} \mathbf{I}$, the likelihood function can be written as

$$
p(\mathbf{g} \mid \boldsymbol{\theta}(k), k)=\frac{1}{\pi^{N}|\varepsilon|^{2 N}} \exp \left(-\frac{1}{|\varepsilon|^{2}}\|\mathbf{g}-\mathbf{R} \boldsymbol{\gamma}(\boldsymbol{\theta}(k))\|^{2}\right) .
$$

It will increase with increasing $k$, since a more complex model fits the observations better. As a consequence, maximization of the likelihood function is not sufficient for model selection. Instead of using only the likelihood as a criterion, penalized likelihood criteria are used for model selection. The general form of penalized likelihood criteria is

$$
\hat{\boldsymbol{\theta}}(k)=\arg \max _{\theta(k)}\{\ln p(\mathbf{g} \mid \boldsymbol{\theta}(k), k)-C(\boldsymbol{\theta}(k))\} .
$$

$\ln p(\mathbf{g} \mid \boldsymbol{\theta}(k), k)$ is the $\log$-likelihood and $C(\boldsymbol{\theta}(k))$ is a complexity penalty, from which we can see that model selection is actually a tradeoff between how well the model fits the data and the complexity of the model. Note that the likelihood depends on the noise model, e.g., for Gaussian noise, the log-likelihood is essentially the sum of squared residuals. If this term only depends on the model dimension, then

$$
\hat{k}=\arg \max _{k}\{\ln p(\mathbf{g} \mid \hat{\boldsymbol{\theta}}(k), k)-C(k)\} .
$$

In other words, estimate the best parameters for each $k$, and then choose among these models. In our application, model

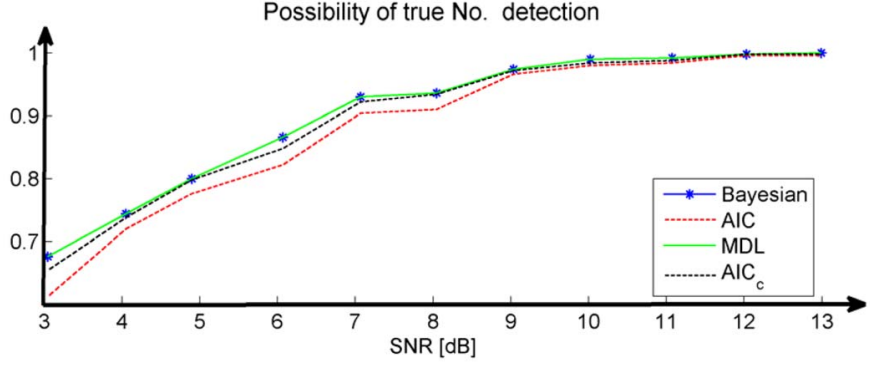

Fig. 7. Probability of correctly detecting two scatterers using different model selection schemes.

complexity only depends on the number of scatterers $n_{p}$ in the azimuth-range pixel. It is common in the literature to multiply the cost function by a factor of minus two

$$
\hat{k}=\arg \min _{k}\{-2 \ln p(\mathbf{g} \mid \hat{\boldsymbol{\theta}}(k), k)+2 C(k)\} .
$$

For each $k=3 n_{p}$ (e.g., $n_{p}=1,2$, or 3 ), the estimated amplitudes, phases, and elevations of the $n_{p}$ scatterers are used to synthesize an estimate of $\gamma$ and to compute $\|\mathbf{g}-\mathbf{R} \hat{\boldsymbol{\gamma}}(\hat{\boldsymbol{\theta}}(k))\|^{2}$, the exponent of the likelihood function. The preferred model is finally the one with the lowest penalized likelihood criterion value according to (15).

There are many types of penalized likelihood criteria, such as the Bayesian information criterion (BIC), the Akaike information criterion (AIC), and the minimum description length (MDL). Their basic principles are the same, and the main difference is in the penalty term. In [30], BIC, MDL, and AIC are discussed in detail for the purpose of determining the number of scatterers inside an azimuth-range pixel of multipass SAR data stack with nine acquisitions.

$B I C$ is also called the Schwarz criterion or Schwarz information criterion (SIC). It is so named because Gideon E. Schwarz [31] gave a Bayesian argument for adopting it. The detailed derivation and performance of BIC are described in [32]. If the models are quasi-nested [33], BIC with $C(k)=0.5 k \ln N$, where $N$ refers to the number of samples (in our case, the number of acquisitions), is an approximation of the Bayesian method that says that models should be compared according to their posterior probabilities.

$M D L$ is a formalization of Occam's razor and tries to find the hypotheses or combination of hypotheses that compress the data the most [34]. The MDL was introduced by Jorma Rissanen in 1978 [35]; it is an important concept in information and learning theory. Without prior knowledge of the model, it is identical to BIC with a penalty term of $C(k)=0.5 k \ln N$.

$A I C$ with $C(k)=k$ tries to minimize the expected relative distance between the fitted model and the unknown true mechanism that generated the observed data [36]. Rejecting a null hypothesis when it should have been accepted creates a type I error; accepting a null hypothesis when it should have been rejected creates a type II error. AIC effectively trades off those two types of errors. As a result, AIC may give less weight to simplicity than to data fit as compared to classical hypothesis testing [37]. Therefore, when the number of samples is large, AIC tends to underpenalize complexity. 


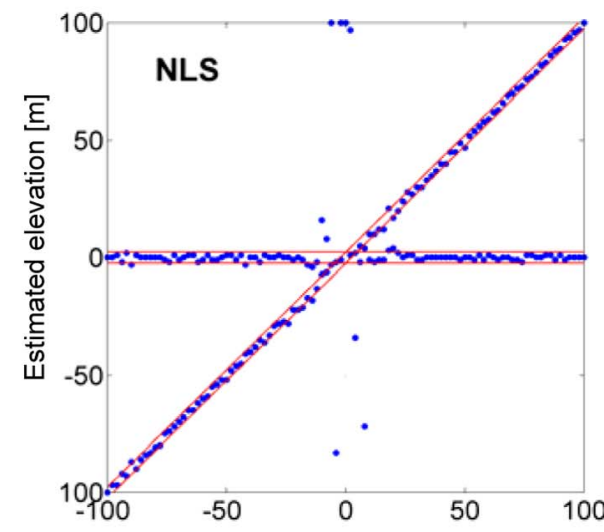

True elevation of the shifting scatterer $[\mathrm{m}]$

(a)

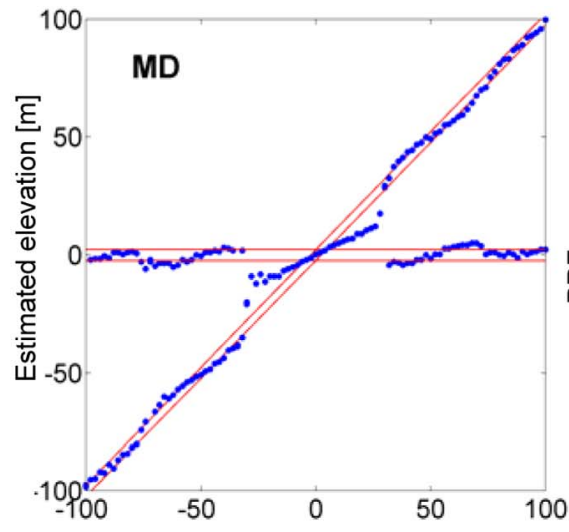

True elevation of the shifting scatterer $[\mathrm{m}]$

(b)

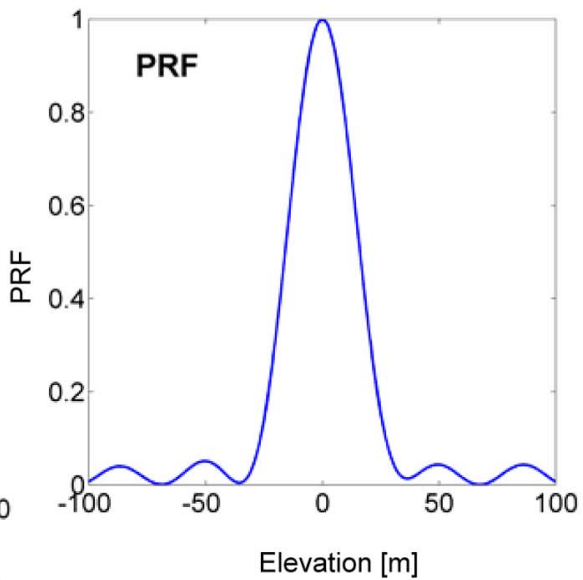

(c)

Fig. 8. Estimated elevations using (a) NLS estimation and (b) MD with SNR $=20 \mathrm{~dB}$. (c) Elevation point spread function from SVD-Wiener reconstruction. The estimation truth is a horizontal line referring to the ground and a diagonal line referring to the scatterer at variable elevation. The red lines in the plots show \pm 3 times the CRLB on elevation estimates for the single scatterer.
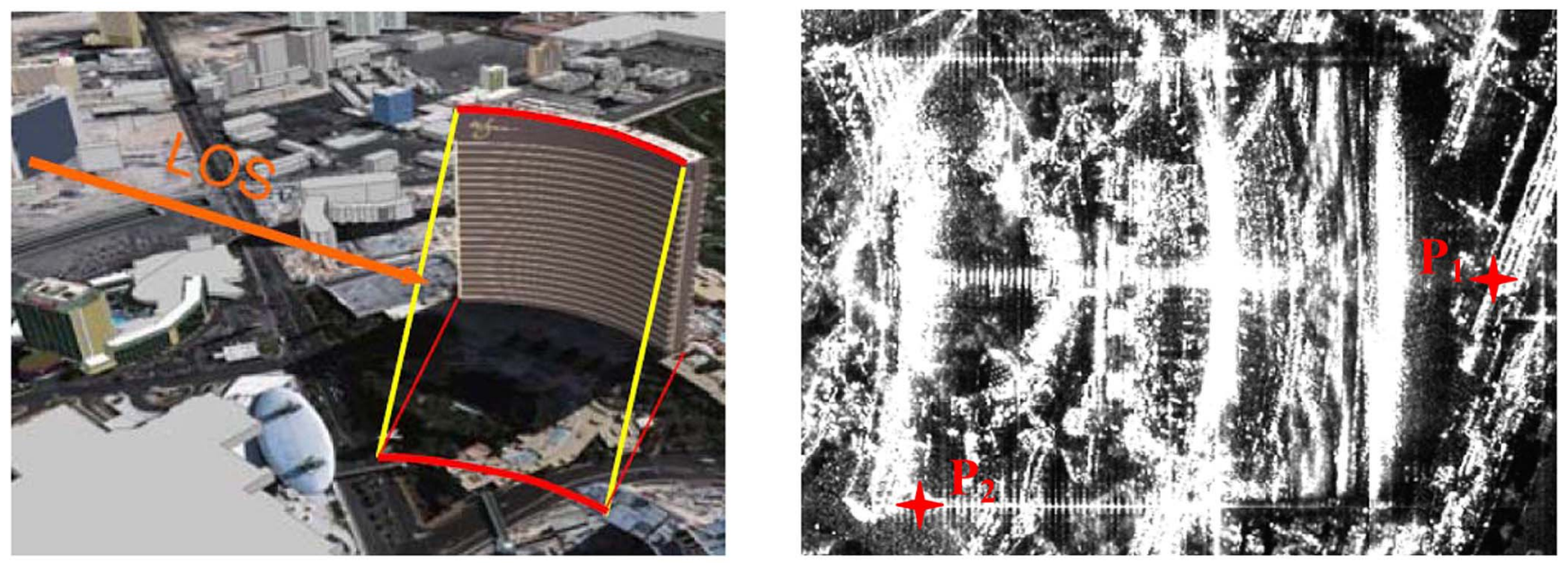

Fig. 9. Wynn hotel, Las Vegas. (Left) Optical image with viewing direction of SAR (LOS = line of sight) and (yellow) two iso-elevation lines. (Right) Mean TerraSAR-X intensity image with (red) analysis points P1 and P2.
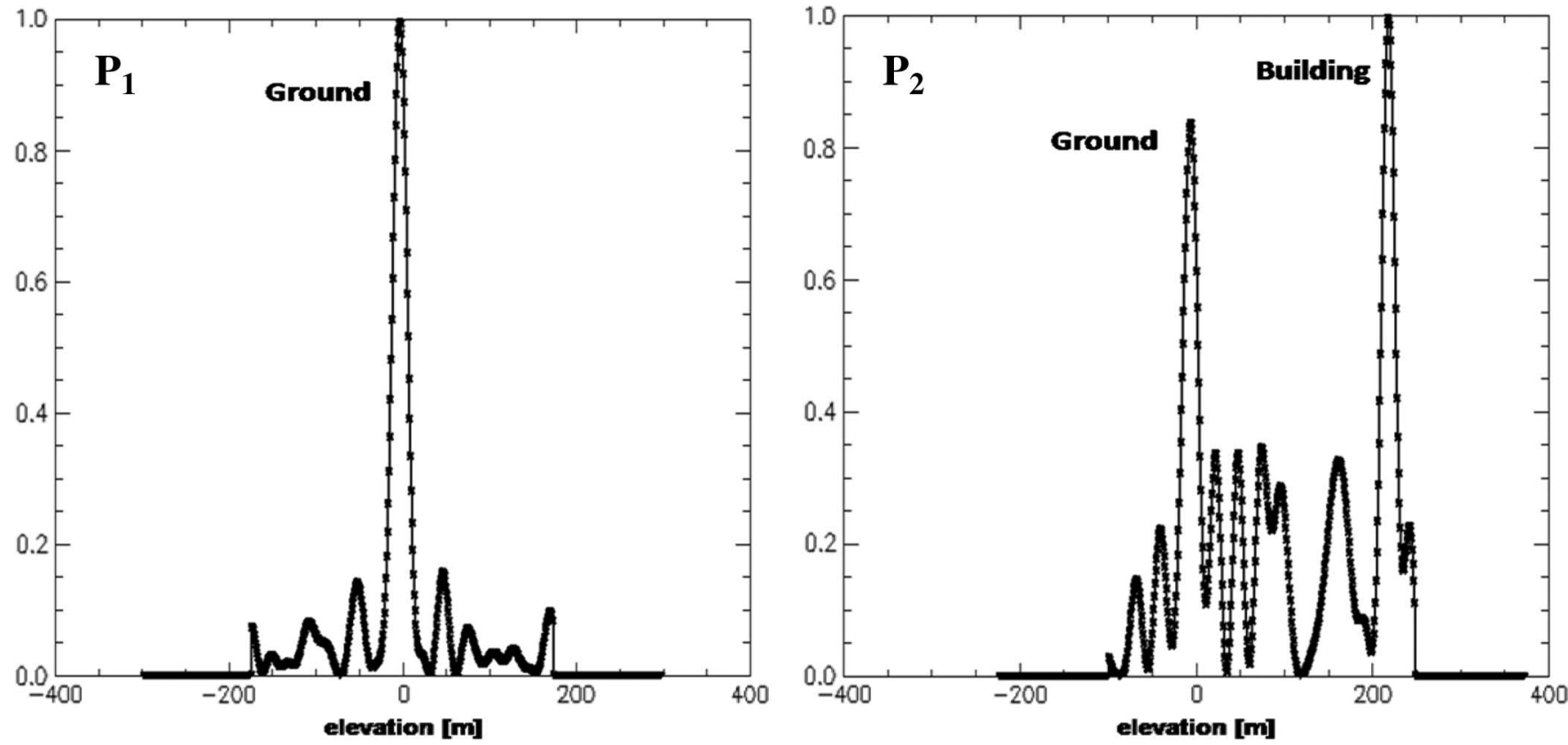

Fig. 10. Elevation profiles at analysis points P1 (single scatterer) and P2 (two scatterers) marked in Fig. 11. 

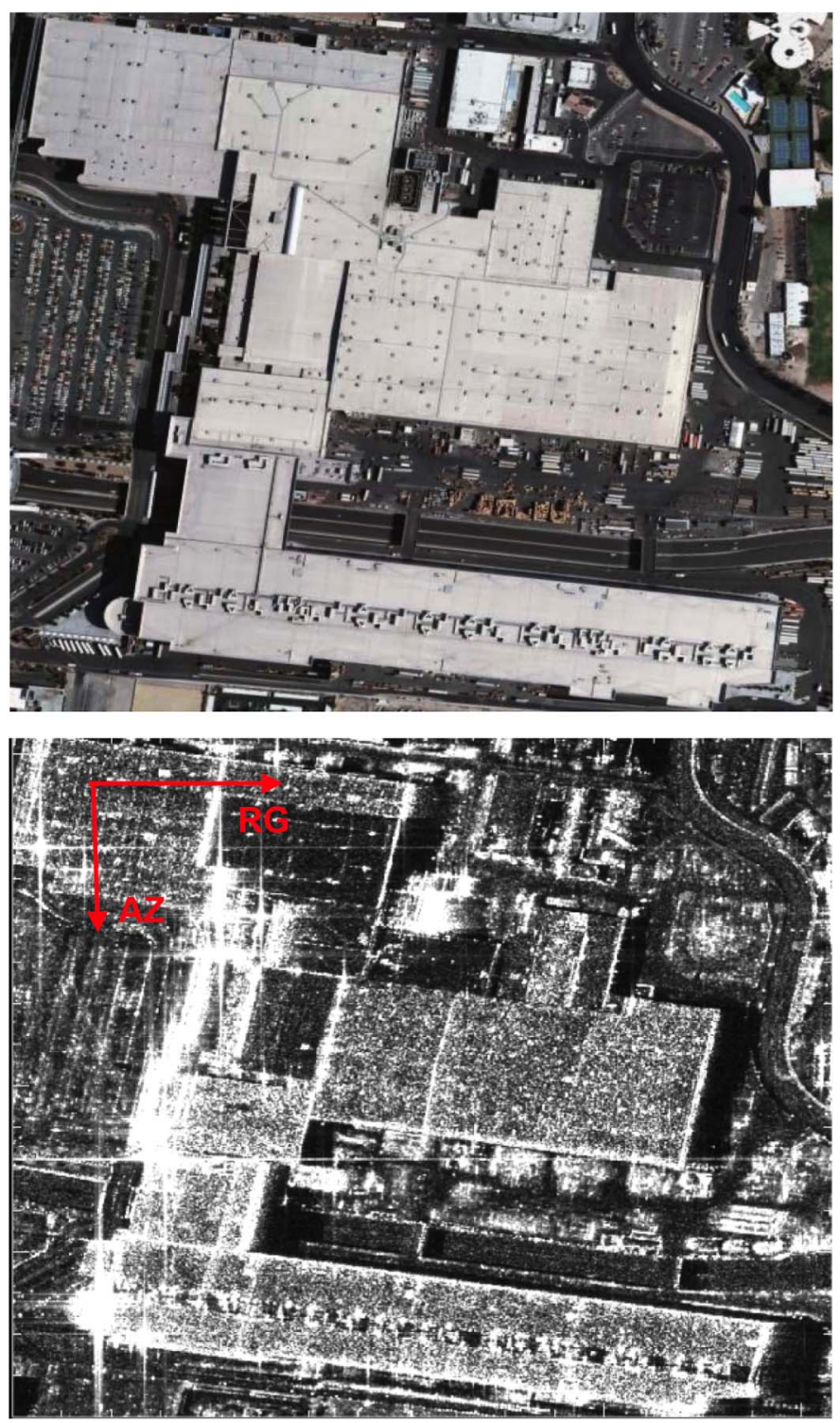

Fig. 11. (Top) Las Vegas Convention Center (Google Earth). (Bottom) TerraSAR-X intensity map.

$A I C c$ is the small-sample bias adjustment for AIC [38]

$$
A I C c=A I C+\frac{2 k(k+1)}{N-k-1} .
$$

An issue with all these model selection methods is selection bias, which cannot be easily corrected. Selection bias refers to the fact that model criteria are particularly risky when a selection is made from a large number of competing models. The random fluctuation in the data will increase the scores of some models more than others. The more models there are, the greater is the risk that the optimal model is chosen at random. In [36], it was emphasized that selection criteria should not be followed blindly and that the term "selection" suggests something definite, which, in fact, has not been reached. Therefore, the selection of a criterion must be based on the experiments for the specific situation, and it is also not possible for model order selection algorithms to control the false-alarm probability.
In order to choose a favorable model selection method, different schemes are evaluated on simulated data with the elevation aperture sampling of Fig. 2. The decorrelation effect is simulated by adding Gaussian noise with a certain SNR. Phase noise due to unmodeled deformation and atmospheric effects are simulated by adding a uniformly distributed phase on $[-0.5 \pi, 0.5 \pi)$. The simulated "truth" is two scatterers at elevations of -20 and $40 \mathrm{~m}$ with reflectivities of 1 and 0.8 , respectively. The distance between the two scatterers is hence 1.5 elevation resolution cells. The most important characteristic for evaluating the performance of the model selection criteria is the detection rate that refers here to the probability of correctly detecting the number of scatterers. A Monte Carlo simulation with 1000 realizations per SNR value was performed to evaluate the detection rates of different schemes. The probability of correctly detecting two scatterers for various SNRs is shown in Fig. 7.

In this example, the number of scatterers is chosen from three hypotheses, namely, one, two, or three scatterers. Since the complexity of the model only depends on $k$, the MDL is identical to BIC in our application, and they give the same results. All the model selection schemes appear to have similar performance. At a typical SNR of $3 \mathrm{~dB}$, a threshold that is also often used for persistent scatterer identification, the probability of correctly detecting the number of scatterers is at least $60 \%$. Overall, the MDL and BIC provide the best performance in our case and are used in the following experiments.

\section{PARAmetric Estimation}

With prior knowledge $n_{p}$, the parameters associated with individual scatterers, such as elevation, reflectivity, and LOS velocity (for D-TomoSAR only), can be estimated by either implementing a parametric estimation method, such as NLS, or simply detecting $n_{p}$ peaks of the nonparametric estimates.

For the following, let us consider that the elevation reflectivity profile is composed of $n_{p}$ delta functions of complex amplitudes $x_{i}$

$$
\gamma(s)=\sum_{i=1}^{n_{p}} x_{i} \cdot \delta\left(s-s_{i}\right)
$$

$N L S$ : The noise-corrupted SAR observations of the $n_{p}$ scatterers is according to the observation equation (4) [39]

$$
g_{n}=\sum_{i=1}^{n_{p}} x_{i} \exp \left(j 2 \pi \xi_{n} s_{i}\right)+v_{n}, \quad n=1, \ldots, N
$$

where $v_{n}$ is the observation noise. As both the complex amplitudes $x_{i}$ and the elevations $s_{i}$ of the individual $n_{p}$ scatterers are unknown, the spectrum estimation problem is nonlinear. Although the signal model is nonlinear, it is at least linear in the amplitude

$$
\mathbf{g}=\mathbf{H}(s) \mathbf{x}+\mathbf{u}
$$



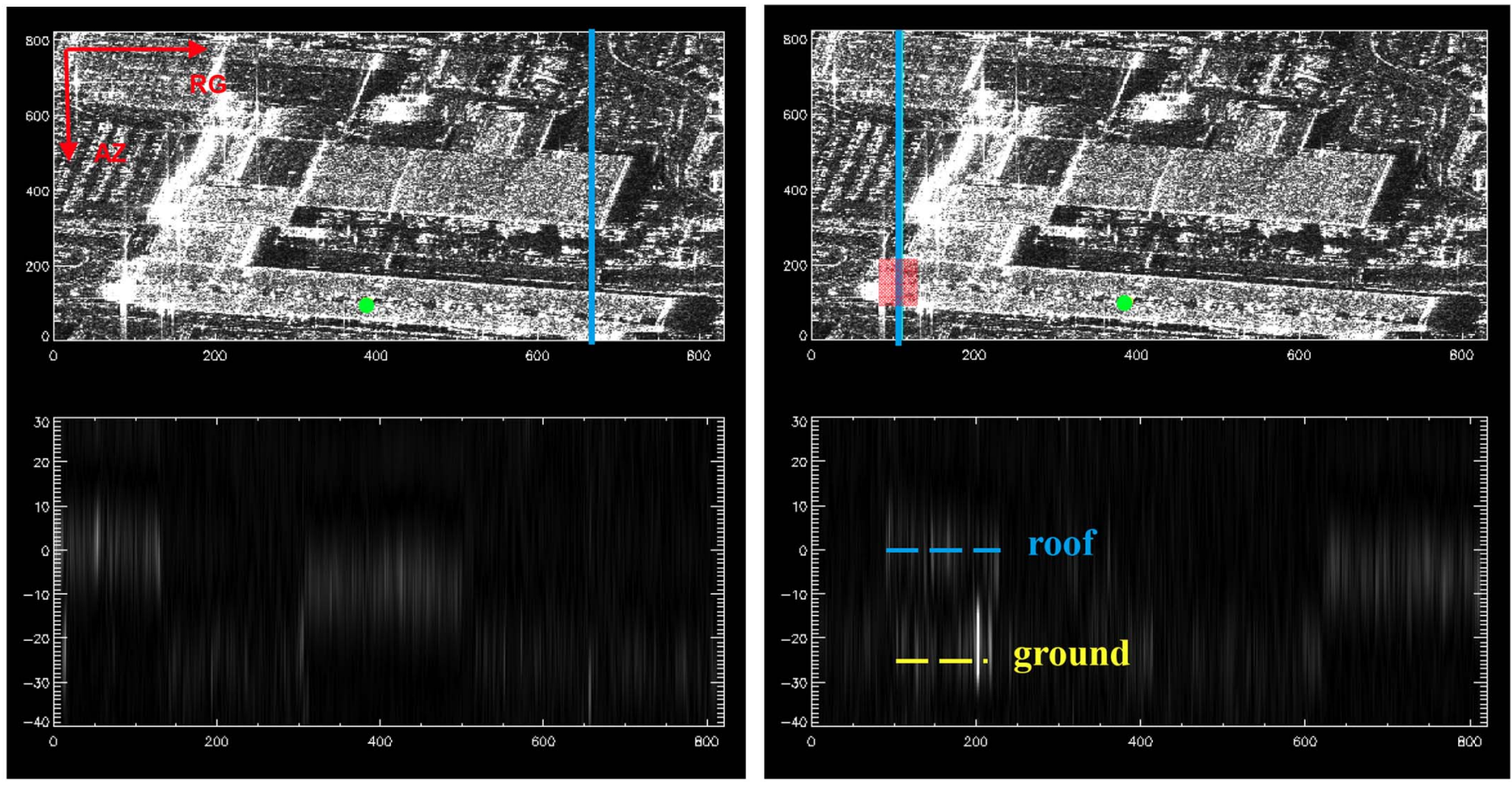

Fig. 12. (Top) TerraSAR-X radar intensity image of the Las Vegas Convention Center. The green dot is the reference point, and the blue lines are the positions of the respective slices. (Bottom) The estimated reflectivity is shown in the azimuth-elevation plane [horizontal: azimuth; vertical: elevation, converted to height (in meters)].

with

$$
\begin{gathered}
\mathbf{g}=\left[\begin{array}{c}
g_{1} \\
\cdot \\
\cdot \\
\cdot \\
g_{N}
\end{array}\right]_{N \times 1} \mathbf{H}(s)=\left[\begin{array}{ccc}
e^{j 2 \pi \xi_{1} s_{1}} & \cdots & e^{j 2 \pi \xi_{1} s_{n_{p}}} \\
\cdot & & \cdot \\
\cdot & & \cdot \\
\dot{e}^{j 2 \pi \xi_{N} s_{1}} & \cdots & e^{j 2 \pi \dot{\xi}_{N} s_{n_{p}}}
\end{array}\right]_{N \times n_{p}} \\
\mathbf{x}=\left[\begin{array}{c}
x_{1} \\
\cdot \\
\cdot \\
\cdot \\
x_{n_{p}}
\end{array}\right]_{n_{p} \times 1} \mathbf{u}=\left[\begin{array}{c}
v_{1} \\
\cdot \\
\cdot \\
\cdot \\
v_{N}
\end{array}\right]_{N \times 1}
\end{gathered}
$$

where the matrix $\mathbf{H}(s)$ depends on the unknown elevations of the scatterers. As this model is linear in amplitude and nonlinear in elevation, the least squares error may be minimized with respect to $\mathbf{x}$ in a closed analytic form and thus be reduced to a function of elevations only, which means that an $n_{p}$-dimensional search is needed (for D-TomoSAR, an additional search for velocity is required). Since the object function [40] x, which minimizes

$$
J(s, \mathbf{x})=(\mathbf{g}-\mathbf{H}(s) \mathbf{x})^{\mathrm{T}}(\mathbf{g}-\mathbf{H}(s) \mathbf{x})
$$

for a given $s$, is

$$
\hat{\boldsymbol{x}}=\left(\mathbf{H}^{\mathrm{T}}(s) \mathbf{H}(s)\right)^{-1} \mathbf{H}^{\mathrm{T}}(s) \mathbf{g}
$$

the resulting error is

$$
J(s, \hat{\boldsymbol{x}})=\mathbf{g}^{\mathrm{T}}\left(\mathbf{I}-\mathbf{H}(s)\left(\mathbf{H}^{\mathrm{T}}(s) \mathbf{H}(s)\right)^{-1} \mathbf{H}^{\mathrm{T}}(s)\right) \mathbf{g} .
$$
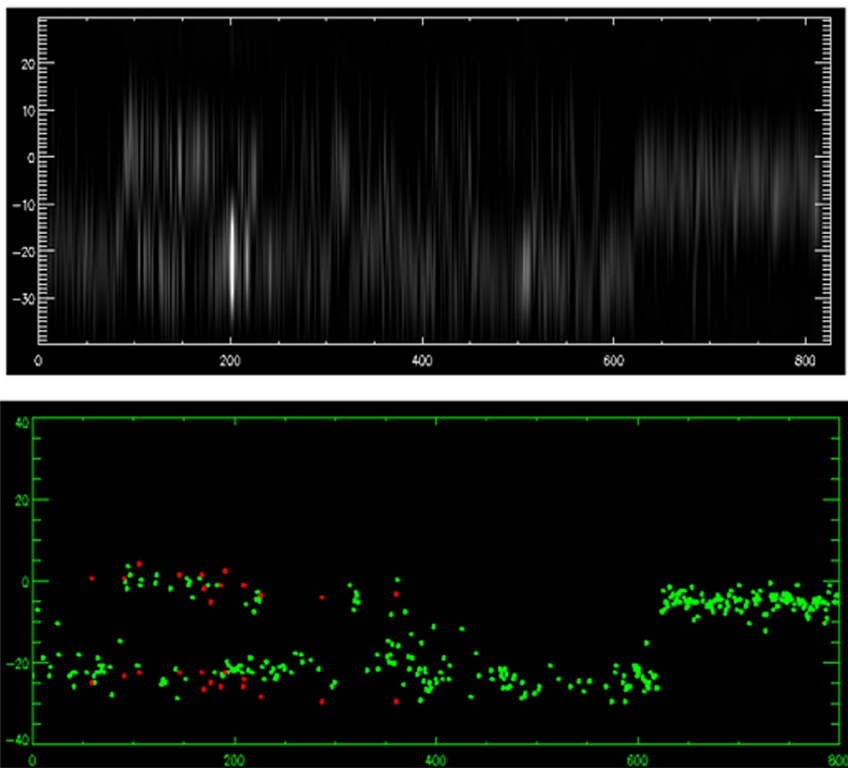

Fig. 13. (Top) Reconstructed reflectivity slice (azimuth-elevation plane) (Bottom) Estimated elevation (converted to height) and model selection results (green dots: single scatterer in an azimuth-range pixel; red dots: two scatterers).

The problem now reduces to a maximization of $\mathbf{g}^{\mathrm{T}} \mathbf{H}(s)\left(\mathbf{H}^{\mathrm{T}}(s) \mathbf{H}(s)\right)^{-1} \mathbf{H}^{\mathrm{T}}(s) \mathbf{g}$ over $n_{p}$ values of $s$, and a grid search can be used. With Gaussian white noise, NLS is identical to MLE. It is therefore theoretically the best estimator for our application if and only if the data closely agree with the assumed model. However, multidimensional search leads to a large computational effort.

Maxima detection (MD): As implied by the name, MD detects $n_{p}$ peaks in the nonparametric estimates. It is relatively 
fast. However, it may introduce estimation bias caused by interference between multiple scatterers, i.e., the location of the peaks may be shifted by the sidelobes of other scatterer responses. In addition, it has the inherent elevation resolution limit of nonparametric spectral estimation and is therefore not capable of separating too close objects.

Two scatterers inside one resolution cell, among which one changes the elevation gradually from -100 to $100 \mathrm{~m}$ and the other stays at zero elevation, are simulated as an example for evaluating the performance of both methods. Fig. 8 shows the estimated elevation of the two scatterers by NLS [Fig. 8(a)] and MD [Fig. 8(b)] with $\mathrm{SNR}=20 \mathrm{~dB}$. The $x$-axis refers to the true elevation of the shifting scatterer. The $y$-axis refers to the estimated elevation. The ideal image should be two straight lines (one horizontal and one diagonal).

The red lines in the plots indicate \pm 3 times the CRLB $\sigma_{\hat{s}}$ on elevation estimates for the single-scatterer case [(3)]. Fig. 8(c) shows the elevation PRF with elevation aperture sampling shown in Fig. 2. As expected, NLS gives the better performance. When the distance between the two scatterers is large enough (low interference effect), NLS is able to locate the scatterers quite well with localization accuracy within the $3 \sigma_{\hat{s}}$ band. Correspondingly, MD of nonparametric estimates is limited by the elevation resolution. Moreover, even with the two scatterers further apart than the resolution cell size, MD suffers from the interference of the scatterers [Fig. 8(b)]. The elevation estimate of one scatterer is systematically biased by the sidelobes of the other and vice versa, even though the SNR is high.

\section{EXPERIMENTAL RESULTS}

\section{A. Las Vegas, Wynn Hotel}

The Las Vegas Wynn hotel has been chosen as a test building to demonstrate the potentials of layover object separation since it is very high and has strong layover effect in the SAR image. Fig. 9 (left) shows the Wynn hotel in Las Vegas with a height close to $200 \mathrm{~m}$, corresponding to an elevation range of $380 \mathrm{~m}$. The right image in Fig. 9 is the corresponding TerraSAR-X intensity image.

Pixels containing multiple scatterers are mainly located at the intersection of the bright texture of the building with structures near the ground. To exemplify the potential of the TomoSAR method, the two pixels marked by red stars have been selected and will be analyzed in the following. As P1 is located outside the region of the high-rise building, it is expected that it only contains a single scatterer situated near the ground. P2 is located at the intersection area; we expect two scatterers inside this pixel, among them one from the ground and one from the building facade. The corresponding reflectivity estimates for those two pixels are shown in the right image of Fig. 10. In this example, we can see the potential of the tomographic approach with TerraSAR-X to separate multiple scatterers in layover areas at a VHR.

\section{B. Las Vegas Convention Center}

The Las Vegas Convention Center is a very interesting test building for 3-D focusing for two reasons. First, it is very big and has a regular shape. Therefore, we are able to check the plausibility of the results. Second, it has a height of about $20 \mathrm{~m}$,
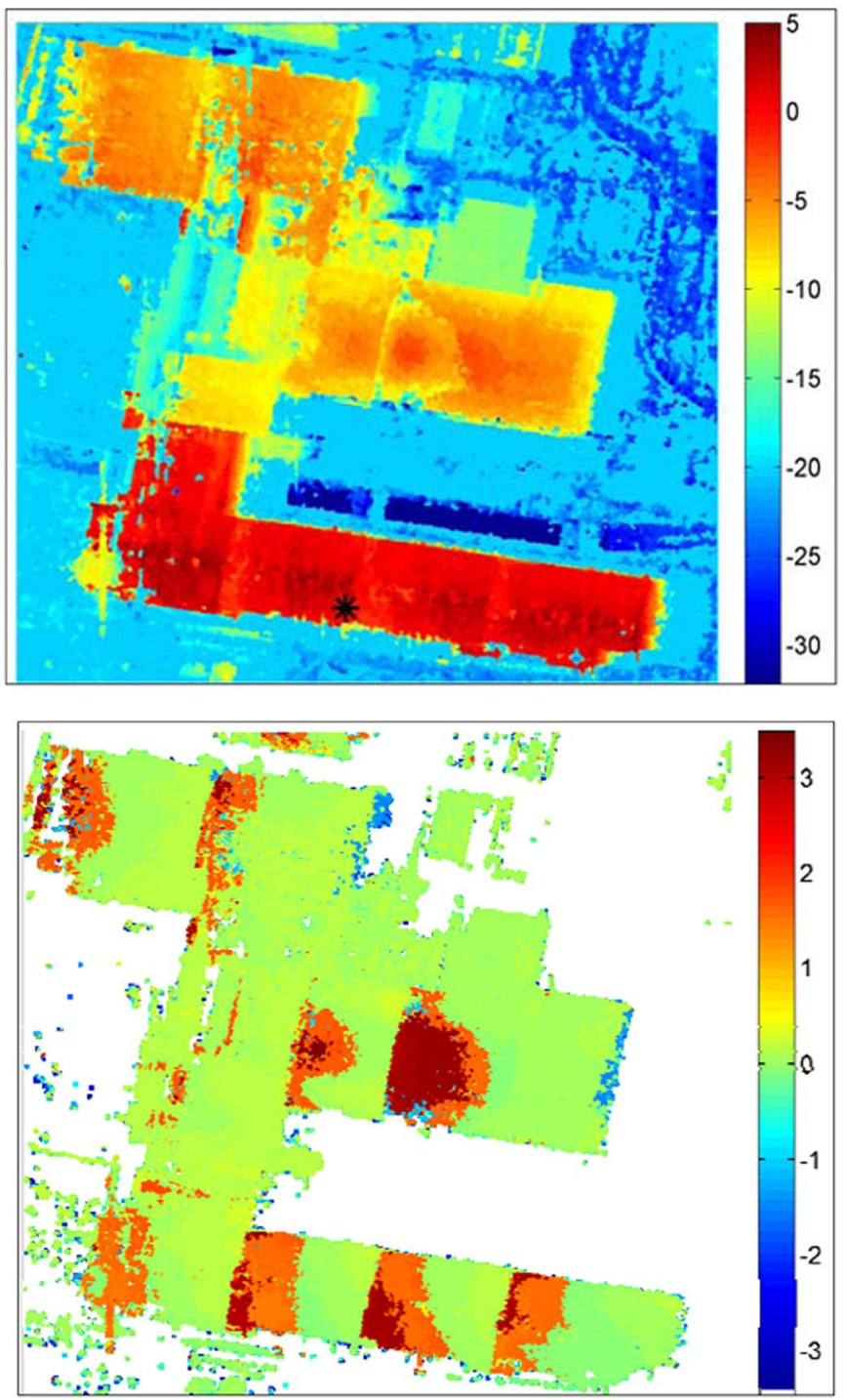

Fig. 14. (Top) Digital surface model of the Las Vegas convention center generated from D-TomoSAR (in meters) (black cross: reference point) and (Bottom) the estimated linear deformation velocity w.r.t. the reference point (in centimeters per year).

the critical distinguishable distance between two scatterers (one from the ground and the other from the building) for our elevation aperture size. The presence of two scatterers within azimuth-range pixels is expected in layover areas. The top image in Fig. 11 shows the convention center visualized in Google Earth. The bottom image is the TerraSAR-X intensity map of the area. After preprocessing, we choose a reference pixel according to [41], which most likely has only a single scatterer inside.

SVD-Wiener, as described in Section IV, is applied to each azimuth-range pixel in the area of interest. Fig. 12 shows an example of the reconstructed reflectivity map of the azimuth-elevation slices with fixed range coordinates marked by bright blue lines. The green dot on the TS-X intensity map is the selected reference that is located on the roof of the convention center. The lower images of Fig. 12 show the reconstructed reflectivity slice (bright means high reflectivity; the $y$-axis refers to the elevation relative to the reference point). The structure of the building can be recognized, and 


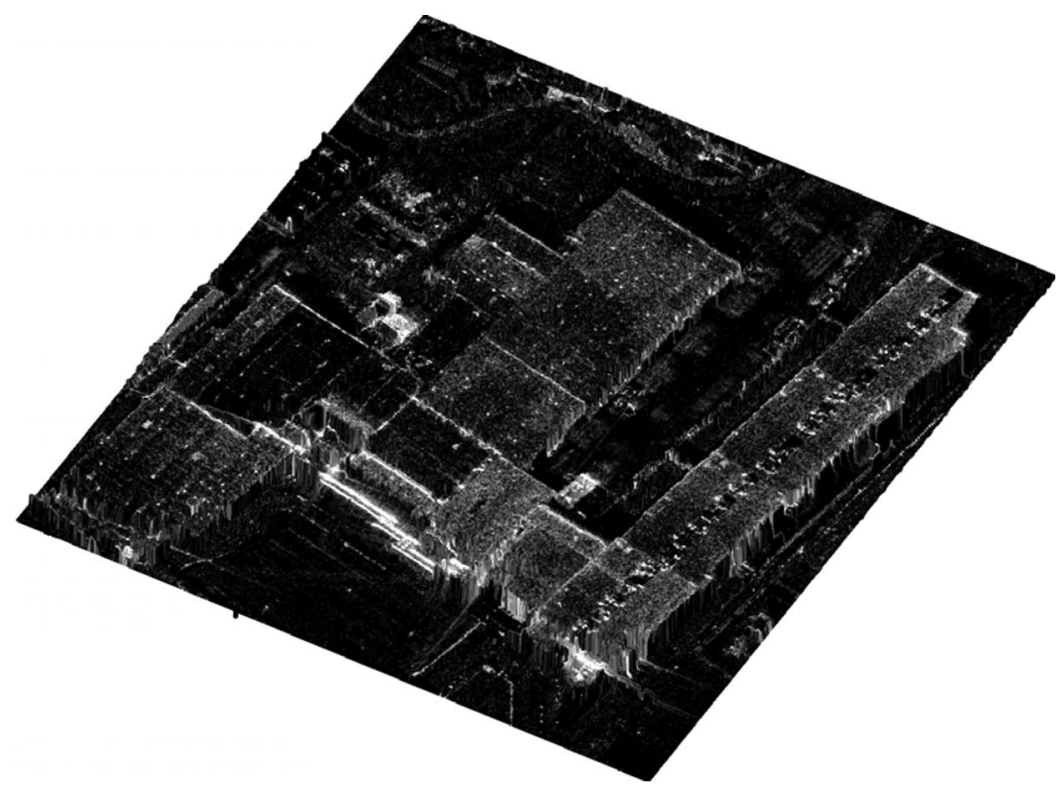

Fig. 15. World at X-band: Tomographic surface reconstruction overlaid by the 3-D reflectivity map.

the reflectivity difference between individual parts can be seen. For instance, from the left slice, it is obvious that the returns from the roof of the building are generally stronger than the returns from the ground and the building has a different height for individual parts (which is not visible in the Google Earth building model). At the range position of the slice on the righthand side, the lower part of the building marked by the red block (left part in the reflectivity slice) is a layover area. From the Google Earth image, we can see that there is a small triangularshaped plaza on the ground made of the same material as the building. Thereby, multiple scatterers are expected. When we check the estimated reflectivity slice, multiple scatterers appear, even though the distance between two scatterers (one from the building marked with a blue line, and the other from the small structures on the ground marked with a yellow line) is approximately at the minimal distinguishable distance. This demonstrates the multiple-scatterer separation potential of TomoSAR and the stability of our algorithm.

After model selection, the number of scatterers inside every azimuth-range pixel is retrieved to provide the required prior knowledge for parameter estimation. The top panel of Fig. 13 shows the reflectivity slice reconstructed by the estimated elevation and reflectivity based on the nonparametric estimates on the right image in Fig. 12. The bottom plot in Fig. 13 reveals the estimated elevations of azimuth-range pixels with good signal. The green dots represent the elevation of a single scatterer in an azimuth-range pixel. The red dots represent the elevation estimates of the detected double scatterers. These results seem plausible. The deformation term is ignored for these results.

From the parametric elevation estimates, a digital surface model of the building can be reconstructed. Even ambiguities in layover areas are resolved. However, deformation has not been considered so far. Therefore, when the deformation signal is strong, the reflectivity reconstruction in the elevation will be distorted, and the elevation estimates degrade. Therefore, we implement D-TomoSAR to our data stack.
Fig. 14 shows the surface model generated from the elevation estimates (converted to height relative to the reference point). The full structure of the convention center has been captured at a very detailed level. Other than the building, more details, such as the roads surrounding the convention center, as well as two bridges above the roads that have weak but correlated returns, are well resolved. Compared to classical interferometric surface reconstruction, TomoSAR overcomes layover and phase-unwrapping problems. The height estimates are very precise compared to the 40.5-m elevation resolution due to the high SNR of TerraSAR-X data. There are still some distortions in the middle of the image where a flat roof surface is expected. It may be due to the incorrect linear deformation model assumption. Since the deformation is presumably caused by thermal dilation, it rather follows a periodic seasonal model. The bottom image of Fig. 14 shows the estimated deformation velocity relative to the reference point. Some areas exhibit a significant uplift of up to $3 \mathrm{~cm} /$ year.

Fig. 15 shows the final surface model with deformation correction overlaid by the reflectivity map. This, for the first time, visualizes in detail how the convention center would look like from the position of TerraSAR-X if our eyes could see $\mathrm{X}$-band radiation. This may lead to a better understanding of the nature of scattering. For instance, an overview about the multiple bounce can be acquired by looking at the very bright structure in Fig. 15. Also, the very bright individual scatterers that behave as corner reflectors can be precisely located. This may help in finding natural corner reflectors.

\section{CONCLUSION}

This paper has demonstrated the potential of the new class of VHR spaceborne SAR systems, like TerraSAR-X and COSMO-Skymed, for TomoSAR in urban environment. A stack of TerraSAR-X high-resolution spotlight data over the city of Las Vegas has been used. Compared to the medium resolution SAR systems available so far, the information content 
and level of detail have increased dramatically. A full tomographic high-resolution reconstruction of a building complex has been presented.

Depending on the application, nonparametric or parametric estimation methods are preferred. Examples for both classes of estimators have been demonstrated and compared to the Cramér-Rao bound. Motivated by the ill-determinedness of the problem, a MAP estimator has been proposed, which leads to a Wiener-type regularization for the nonparametric SVD method for both 3-D and 4-D (differential) tomographic reconstructions. Model selection, i.e., the estimation of the number of discrete scatterers in a resolution cell, has been shown to be a necessary prerequisite for parametric estimation.

One of the major error sources is unmodeled, e.g., nonlinear, motion. These phase errors are able to deteriorate the elevation estimates. With the launch of TanDEM-X, singlepass (motion-free) data pairs will be available. Mixed data stacks from TerraSAR-X and TanDEM-X will be an attractive option for mitigating motion errors and for retrieving profiles of temporarily decorrelated scatterers.

\section{ACKNOWLEDGMENT}

The authors would like to thank N. Adam of DLR for supporting the data preprocessing.

\section{REFERENCES}

[1] A. Reigber and A. Moreira, "First demonstration of airborne SAR tomography using multibaseline L-band data," IEEE Trans. Geosci. Remote Sens., vol. 38, no. 5, pp. 2142-2152, Sep. 2000.

[2] F. Lombardini, "Differential tomography: A new framework for SAR interferometry," in Proc. IGARSS, Toulouse, France, 2003, pp. 1206-1208.

[3] P. Pasquali, C. Prati, F. Rocca, and M. Seymour, "A 3-D SAR experiment with EMSL data," in Proc. IGARSS, 1995, pp. 784-786.

[4] S. Sauer, L. Ferro-Famil, A. Reigber, and E. Pottier, "3D urban remote sensing using dual-baseline POL-InSAR images at L-band," in Proc. IGARSS, 2008, pp. IV-145-IV-148.

[5] J. Homer, D. I. Longstaff, Z. She, and D. Gray, "High resolution 3-D imaging via multi-pass SAR," Proc. Inst. Elect. Eng.-Pt. F, vol. 149, no. 1, pp. 45-50, Feb. 2002

[6] Z. She, D. Gray, R. E. Bogner, J. Homer, and D. Longstaff, "Three-dimensional spaceborne synthetic aperture radar (SAR) imaging with multipass processing," Int. J. Remote Sens., vol. 23, no. 20, pp. 43574382, Oct. 2002

[7] G. Fornaro, F. Serafino, and F. Lombardini, "Three-dimensional multipass SAR focusing: Experiments with long-term spaceborne data," IEEE Trans. Geosci. Remote Sens., vol. 43, no. 4, pp. 702-714, Apr. 2005.

[8] X. Zhu, N. Adam, and R. Bamler, "First demonstration of spaceborne high resolution SAR tomography in urban environment using TerraSAR-X data," in Proc. CEOS SAR Workshop Calibration Validation, 2008.

[9] G. Fornaro and F. Serafino, "Imaging of single and double scatterers in urban areas via SAR tomography," IEEE Trans. Geosci. Remote Sens., vol. 44, no. 12, pp. 3497-3505, Dec. 2006.

[10] G. Fornaro, D. Reale, and F. Serafino, "Four-dimensional SAR imaging for height estimation and monitoring of single and double scatterers," IEEE Trans. Geosci. Remote Sens., vol. 47, no. 12, pp. 224-237, Dec. 2009.

[11] P. C. Hansen, Regularization Tools, 1992. [Online]. Available: http:// www.imm.dtu.dk/ pch/Regutools/

[12] M. Eineder, N. Adam, R. Bamler, and N. Yague-Martinez, "Spaceborne spotlight SAR interferometry with TerraSAR-X," IEEE Trans. Geosci. Remote Sens., vol. 47, no. 5, pp. 1524-1535, May 2009.

[13] S. D'Amico, C. Arbinger, M. Kirschner, and S. Campagnola, "Generation of an optimum target trajectory for the TerraSAR-X repeat observation satellite," in Proc. 18th Int. Symp. Space Flight Dynamics, Munich, Germany, 2004, p. 137.
[14] R. Bamler, M. Eineder, N. Adam, X. Zhu, and S. Gernhardt, "Interferometric potential of high resolution spaceborne SAR," Photogramm. Fernerkundung Geoinf., vol. 2009, no. 5, pp. 407-419, Nov. 2009.

[15] S. Buckreuss, private communication, Aug. 31, 2009.

[16] G. Fornaro, F. Serafino, and F. Soldovieri, "Three-dimensional focusing with multipass SAR data," IEEE Trans. Geosci. Remote Sens., vol. 41, no. 3, pp. 507-517, Mar. 2003.

[17] B. Kampes, Radar Interferometry, The Persistent Scatterer Technique, 1st ed. New York: Springer-Verlag, 2006, ser. Remote Sensing and Digital Image Processing Series.

[18] F. Meyer, S. Gernhardt, and N. Adam, "Long-term and seasonal subsidence rates in urban areas from persistent scatterer interferometry," in Proc. URBAN, Paris, France, 2007.

[19] C. Cafforio, C. Prati, and F. Rocca, "SAR data focusing using seismic migration techniques," IEEE Trans. Aerosp. Electron. Syst., vol. 27, no. 2 , pp. 194-207, Mar. 1991.

[20] N. Adam, B. Kampes, M. Eineder, J. Worawattanamateekul, and M. Kircher, "The development of a scientific permanent scatterer system," presented at the Int. Society Photogrammetry Remote Sensing (ISPRS) Hannover Workshop, Hannover, Germany, 2003.

[21] F. Lombardini and A. Reigber, "Adaptive spectral estimation for multibaseline SAR tomography with airborne L-band data," in Proc. IEEE IGARSS, 2003, vol. 3, pp. 2014-2016.

[22] S. Guillaso and A. Reigber, "Polarimetric SAR Tomography (POLTOMSAR)," in Proc. POLINSAR, Frascati, Italy, 2005.

[23] F. Gini and F. Lombardini, "Multibaseline cross-track SAR interferometry: A signal processing perspective," IEEE Aerosp. Electron. Syst. Mag., vol. 20, no. 8, pp. 71-93, Aug. 2005.

[24] F. Lombardini and M. Pardini, "Detection of scatterer multiplicity in spaceborne SAR tomography with array errors," in Proc. IEEE Radar Conf., May 4-8, 2009, pp. 1-6.

[25] P. C. Hansen, "The truncated SVD as a method for regularization," BIT Numer. Math., vol. 27, no. 4, pp. 534-553, Oct. 1987.

[26] G. H. Golub, V. Klema, and G. W. Stewart, "Rank degeneracy and least squares problems," Dept. Comput. Sci., Univ. Maryland, College Park, MD, Tech. Rep. TR-456, 1976.

[27] H. P. William, T. A. Saul, V. T. William, and P. F. Brian, Numerical Recipes in $C$, 2nd ed. New York: Press Syndicate Univ. Cambridge, 1992.

[28] X. Zhu, N. Adam, and R. Bamler, "Space-borne high resolution tomographic interferometry," in Proc. IEEE IGARSS, Cape Town, Africa, 2009, pp. IV-869-IV-872.

[29] F. Lombardini and F. Gini, "Model order selection in multi-baseline interferometric radar systems," EURASIP J. Appl. Signal Process., vol. 2005, no. 20, pp. 3206-3219, 2005.

[30] X. Zhu, "High-resolution spaceborne radar tomography," M.S. thesis, Lehrstuhl für Photogrammetrie und Fernerkundung, Tech. Univ. München, Lehrstuhlbibliothek, München, Germany, 2008.

[31] G. Schwarz, "Estimating the dimension of a model," Ann. Statist., vol. 6, no. 2, pp. 461-464, Mar. 1978

[32] K. P. Burnham and D. R. Anderson, "Multimodel inference: Understanding AIC and BIC in model selection," Sociol. Methods Res., vol. 33, no. 2, pp. 261-304, Nov. 2004.

[33] M. R. Forster, "Key concepts in model selection: Performance and generalizability," J. Math. Psychol., vol. 44, no. 1, pp. 205-231, Mar. 2000.

[34] J. Rissanen, "A short introduction to Model Selection," Kolmogorov Complexity and Minimum Description Length, 2005. [Online]. Available: www.mdl-research.org

[35] J. Rissanen, "Modelling by shortest data description," Automatica, vol. 14 , no. 5, pp. 465-471, Sep. 1978.

[36] M. Browne, "Cross-validation methods," J. Math. Psychol., vol. 44, no. 1, pp. 108-132, Mar. 2000.

[37] Y. Sakamoto, M. Ishiguro, and G. Kitagawa, Akaike Information Criterion Statistics. Dordrecht, The Netherlands: Reidel, 1986.

[38] Y. Sakamoto, Categorical Data Analysis by AIC. Tokyo, Japan: KTK Scientific Publishers, 1991.

[39] X. Zhu, N. Adam, R. Brcic, and R. Bamler, "Space-borne high resolution SAR tomography: Experiments in urban environment using TerraSAR-X data," in Proc. JURSE, 2009.

[40] S. M. Kay, Fundamentals of Statistical Signal Processing. Englewood Cliffs, NJ: Prentice-Hall, 1993.

[41] N. Adam, R. Bamler, M. Eineder, and B. Kampes, "Parametric estimation and model selection based on amplitude-only data in PS-interferometry," presented at the FRINGE Workshop, Frascati, Italy, 2005. CD-ROM 


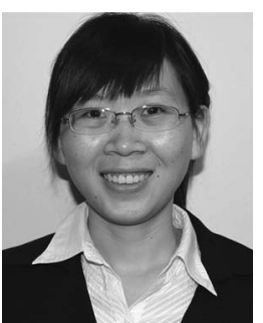

Xiao Xiang Zhu (S'10) was born in Changsha, China, on December 12, 1984. She received the B.S. degree in space engineering from the National University of Defense Technology, Changsha, in 2006 and the M.Sc. degree in earth oriented space science and technology (ESPACE) from the Technische Universität München (TUM), München, Germany, in 2008. She is curently working toward the Ph.D. degree in differential synthetic aperture radar (SAR) tomography using TerraSAR-X data at the Remote Sensing Technology, TUM.

Since May 2008, she has been a Full-Time Scientific Collaborator with Remote Sensing Technology, TUM. Her work is part of the project team "Dynamic Earth," which was established in the International Graduate School of Science and Engineering, TUM, as a result of the German Excellence Initiative in 2007. In October/November 2009, she was a Guest Scientist with the Institute for Electromagnetic Sensing of the Environment (IREA), Italian National Research Council (CNR), Naples, Italy. Her main research interests are in the signal processing field with applications to spaceborne SAR data, in particular SAR tomography, and differential SAR tomography.

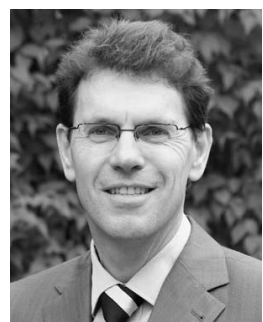

Richard Bamler (M'95-SM'00-F'05) received the Diploma degree in electrical engineering, the Doctor of Engineering degree, and the "Habilitation" degree in signal and systems theory from the Technische Universität München (TUM), München, Germany, in 1980, 1986, and 1988, respectively.

During 1981 and 1989, he was with TUM, working on optical signal processing, holography, wave propagation, and tomography. In 1989, he joined the German Aerospace Center (DLR), Oberpfaffenhofen, Germany, where he is currently the Director of the Remote Sensing Technology Institute (IMF). Since then, he and his team have been working on synthetic aperture radar (SAR) signal processing algorithms (ERS, SIR-C/X-SAR, Radarsat, SRTM, ASAR, TerraSAR-X, and TanDEM-X), SAR calibration and product validation, SAR interferometry, phase unwrapping, estimation theory and model-based inversion methods for atmospheric sounding (GOME, SCIAMACHY, MIPAS, and GOME-2), and oceanography. In early 1994, he was a Visiting Scientist with the Jet Propulsion Laboratory in preparation of the SIC-C/X-SAR missions, and in 1996, he was a Guest Professor with the University of Innsbruck, Innsbruck, Austria. Since 2003, he has been a Full Professor in remote sensing technology with TUM. His current research interests are in algorithms for optimum information extraction from remote sensing data with emphasis on SAR, SAR interferometry, persistent scatterer interferometry, SAR tomography, and ground moving target indication for security-related applications. He and his team have developed and are currently developing the operational processor systems for the German missions TerraSAR-X, TanDEM-X, and EnMAP. He is the author of more than 160 scientific publications, among them about 40 journal papers, a book on multidimensional linear systems theory, and several patents on SAR signal processing. 